\title{
Comparative Functional Analysis of the Anatomy of the appendicular skeleton in two reptilian species
}

\author{
Ali G. Gadel-Rab ${ }^{1}$, Fatma A. Mahmoud ${ }^{2}$, Samy A. Saber $^{3}$, Boshra A. EISalkh ${ }^{4}$, Asmaa A. \\ El-Dahshan ${ }^{4}$, Doaa I. Gewily ${ }^{4}$ \\ 1-Department of Zoology, Faculty of Science, Al-Azhar University, Assiut, Egypt \\ 2-Department of Zoology, Faculty of Science, Assuit University, Assiut, Egypt \\ 3-Department of Zoology, Faculty of Science (Boys branch), Al-Azhar University, Cairo, Egypt \\ 4-Department of Zoology, Faculty of Science (Girls branch), Al-Azhar University, Cairo, Egypt
}

\section{Abstract:}

Corresponding author: Ali Gamal,email: Aligama1200992@yahoo.com

Aim of the work: the present study aimed to illustrate the osteological characters of the appendicular skeleton of two Egyptian lizard species, Acanthodactylus boskianus and Ptyodactylus guttatus and clarify the relationship between characteristics of the appendicular skeleton and mode of their locomotion of the two species. A. boskianus is diurnal insectivorous lizard forage in open area and it is a generalized ground-dwelling species and $P$. guttatus is a diurno-nocturnal omnivorous gecko which is a highly specialized climbing on vertical substrates. Material and methods: in the present study we used Alizarin red and Alcian blue as double skeletal staining to investigate the the bony and cartilaginous pattern of limbs and girdles of A. boskianus and P. guttatus. Results: in P. guttatus, the pectoral girdle was characterized by the presence of the interclavicles was dagger in shape and partially fused with clavicles, while it was cruciform and completely fused with the clavicles in A. boskianus. In P. guttatus, the epicoraoid was well developed, broad bony plates and fused with the interclavicles, while in A. boskianus it was narrow cartilaginous, plates and overlap in the mid-line. Moreover, in P. gutattus two fenestrae were appeared within coracoid and scapula with un-fenestrated cartilaginous sternum, while one fenestra appeared in both coracoid and sternum of $A$. boskianus. On other hand, the pelvic girdle of $P$. guttatus had complete fusions between the pubis, ischium and ilium, while in A. boskianus the pubis articulated poster-omedially with the ischium. In both $P$. gutattus and A. boskianus, the forelimbs posture was in horizontal plane, while the hind limbs were in vertical plane. In $P$. gutattus both the fore and hind limbs were relatively equal in length, while the fore limb in A. boskianus was greatly shorter than the hind limb. Conclusion: the P. gutattus is considered as a one of climbers species which have stout appendicular skeleton to support the climbing process on the vertical surface with relatively short limbs, while A. Boskianus is ground-dwellers and also good climbers and use their claws to walk and climbing on rocky areas and these abilities of locomotion depend on the flexibility of skeletal elements of its appendicular skeleton.

Keywords: girdles, limbs, double stain, reptiles

\section{Introduction}

Locomotion is considered as one of the major functions within the ecology of an animal because of its importance during activities such as feeding, social interactions and predator avoidance [1]. Many animals move through complex and heterogeneous physical environments at varying speeds and using different modes of locomotion ${ }^{[2]}$. Among reptiles, lizards 
have the widest geographical distribution encompassing a wide range of locomotor habitats. This is reflected in a large morphological diversity of the general body form and of the locomotory apparatus among these animals. The interspecific variation of modes of locomotion among lizards has been explored by different authors. Nerveless, have failed to find clear correlations between limb morphology and lifestyle. Thus, lizards are an interesting group to study the effects of radical changes in locomotor habitat (e.g. climbing, swimming or digging vs running) on the locomotory apparatus ${ }^{[3-4]}$.

The present study aimed to clarify the correlation between the morphoosteological characteristics of the appendicular skeleton of two lizard species and mode of their locomotion. The two selected species sharing the same desert habitat, but differ in mode of their locomation; A. boskianus is diurnal insectivorous lizard forage in open area and it is a generalized ground-dwelling species and $P$. guttatus is a diurnonocturnal omnivorous gecko and it is a highly specialized climbing on vertical substrates.

\section{Material and methods}

Fourteen adult Bosc's fringe-toed lizard, Acanthodactylus boskianus and spotted fan-toed geckos, Ptyodactylus guttatus were collected from different regions of Sinai and sacrificed by ether anesthesia. The skeletal elements of three specimens were carefully dissected from the surrounding muscles and fascia and then they were bleached with hydrogen peroxide (H2O2). The figures of appendicular skeleton of both studied specimens were prepared by outlining the anatomical preparations with help of the camera Lucida. To illustrate the bony and cartilaginous pattern of limbs and girdles of both studied specimens, three specimens from each species were stained in a solution of $80 \mathrm{ml}$ ethanol (95\%), 20 $\mathrm{ml}$ glacial acetic acid and $15 \mathrm{mg}$ Alcian blue $8 \mathrm{GN}$ for $24 \mathrm{~h}$, then hydrated in descending series of ethyl alcohol and in water for a period of 7 days with several changes. The soft tissue was completely re-stained and the blue cartilage became discernable from the other tissue after maceration in weak $0.1 \mathrm{KOH}$. The specimens were transferred to $0.1 \% \mathrm{KOH}$ containing $0.1 \%$ Alizarin red-S to turn the solution deep purple, stained for $24 \mathrm{~h}$, until the shafts of the thickest long bones become uniformly deep red. Then the specimens were transferred to a series of $0.1 \% \mathrm{KOH}$ and glycerin $(3: 1,1: 1,1: 3)$, followed by fully glycerin, for approximately $24 \mathrm{~h}$ in each step. Few crystals of thymol were added to pure glycerin to inhibit mold and bacteria, after which the specimens were stored in a cool and dark place. The stained samples were examined and photographed under Leica Sterio microscope.

\section{Results:}

Anatomical investigation of appendicular skeleton

The basic pattern of the skeletal structure of pectoral and pelvic girdles as well as the fore and hind limbs elements ars similar in both studied reptile species; P. guttatus and A. boskianus. At the same time, there were gradual variations in the shape and ossification of some elements with presence or absence of fenestrae as well as the length of the elements constituting the fore and hind limbs and angle of articulation with the pectoral and pelvic girdle. The pectoral girdle of $P$. guttatus and A. boskianus consisted of 


\section{Comparative Functional Analysis of the Anatomy......}

paired elements (clavicles, scapulae, coracoids epicoracoids and suprascapulae) and unpaired element (interclavicles). In $P$. guttatus and $A$. boskianus, the paired clavicle (CL) was completely bony hooklet-shaped structure slightly dilated laterally. The two halves of clavicle were jointed at mid-ventral line, medial to the scapula and articulated laterally with anteroventral notch of the supra-scapula (SSC) (Figs. 1a, 1b, 1c, 1d). Interclavicle (ICL) was unpaired bony structure found in mid-ventral line to joins both halves of the girdle across the midline. In $P$. guttatus, the interclavicles was dagger in shape and partially fused with clavicles, while it was cruciform and completely fused with the clavicles in A. boskianus (Figs.1a,1b,1c,1d). The coracoid (CO) of $P$. guttatus and A. boskianus is a stout triangular paired bony element. The two coracoids are fused antero-dorso-laterally with scapula and articulated medially with the epicoracoid and laterally with the head of humorous bone. In $P$. guttatus, the coracoid and scapulocoracoid fenestrae were observed while in A. Boskianus, only one coracoid fenestra is present (Figs.1a,1b,1c,1d).The epicoraoid (EPC) of both studied species was paired, triangular-shaped flat plate with convexly curved at their inner border. In $P$. guttatus, the epicoraoid was well developed, broad bony plates which were fused medially with the interclavicles. While in $A$. boskianus, the epicoracoid was cartilaginous, narrow plates and overlap in the mid-line (the left being ventral to the right) (Figs.1a, 1b, 1c, 1d). The sternum (ST) of both P. guttatus and A. boskianus was unpaired, partially calcified triangular-shaped structure. In $P$. guttatus, sternum was un-fenestrated while, in A. Boskianus it was fenestrated. The postero-lateral edge of sternum was provided with articulated facets for articulation with ribs. In both $P$. guttatus and $A$. boskianus, the ribs of the ninth, tenth and eleventh vertebrae reach the sternum independently (STR), while ribs of the twelfth and thirteenth vertebrae join to form the xiphisternum (XST) (Figs.1a,1b,1c,1d). The skeleton of fore limb of both studied species which consisted of humerus, radius, ulna, metacarpals and digits were calcified.In P. guttatus and A. boskianus both the proximal and distal ends of the humerus were greatly expanded and flattened with the middle part of humerus in P. guttatus was more or less slender. The proximal end articulated with the glenoid cavity (G) of the pectoral girdle (Figs.1a, 1b,1c,1d). While, the distal end had two heads; one head articulated with the radius, while the other articulates with the ulna (Fig.3).In both P. guttatus and A. boskianus the radius and ulna were separate bones and slightly smaller in length than the humerus (Figs.3,4a,4b,4c,Ad,4e,4f,4g,4h).

The carpals of both studied species which articulate with the radius and ulna, the elements consisted of proximal row of the carpus which was composed of radiale (RLE), centrale (C), ulnare (ULE) and a row of five distal carpals. The radiale was a transverse longer bone of the carpus which articulated postero-laterally with the epiphysis of the radius and articulates anteriorly with the first distal carpus. The ulnare was a rectangular-shaped process that slightly larger than the radiale articulated postero-laterally with the epiphysis of the ulna, anteriorly with the fourth and fifth distal carpals and laterally with the partially calcified pisiform (PIS). The radiale was completely separated from the ulnare by central (Figs.4a, 4b, 4c, Ad, 4e, 4f, 4g, 4h).

The palmar sesamoids (PS) in $A$. boskianus were two semi-triangular 
calcified rudiments which were located on the ventral surface of the proximal phalanges of the digits III and IV (Fig.4g, h). Those sesamoids were not evident in P. guttatus. Moreover, in P. guttatus a small calcified pisifom (PIS) laid on the outer side of the ulnare (Figs.4c, Ad, 4g, 4h). The pentadactyl fore limb of $P$. guttatus and $A$. boskianus was formed of five metacarpals, each of which articulates with one of five phalanges. The phalangeal formula of both $P$. guttatus and $A$. boskianus in each digit from the thumb outward was commonly 2: 3: 4: 5: 3 (Figs. 3, 4a, 4b, 4c, Ad ,4e, 4f, 4g, 4h).

In $P$. guttatus and A. boskianus, the pelvic girdle was composed of two halves, each half of the pelvic girdle consisted of the pubis (P), ilium (IL) and ischium (IS); they met at the acetabulum (AC) and built together an extensive foramen (ischio-pubic fenestra, $\mathrm{Fc}$ ) which was normally undivided. A cartilaignous ischiadic process (spine Ischia, IP) was developed from the ischium and the prepubic (pectinael, PP) process from the posterolateral margin of the pubis. There was a distinct variation in the shape of pubis, ischium and ilium of the two studied species (Figs. 2a, 2b, 2c, 2d).

In $P$. guttatus and A. boskianus, the pubis (P) was a paired ventro-lateral bony process. In $P$. guttatus, the pubis appeared as broad transverse bone fused at ventromidline through partially calcified epipubic process (Ep). While, in $A$. boskianus, the paired pubis seemed as elongated bone with narrow anterior end that articulates at ventro-midline with narrow triangular epipubic process (Ep). Posteriorly, the pubis of both studied species became broad and beared obturator foramen (OF) posterior to prepubic (pectineal process, PP) which was located on the posterolateral margin of pubis. That pectineal process was more developed in $P$. guttatus than that of A. boskianus (Figs. 2a, 2b, 2c, 2d). Moreover, in $P$. guttatus the posterior end of pubis was completely fused posteroventrally with ischium and posterodorsally with ilium, while in $A$. boskianus the pubis articulates posteromedially with the ischium.The ischium (IS) of $P$. guttatus and $A$. boskianus was a paired broad bony element which was completely fused antero-ventrally with the pubis and postero-dorsally with ilium. In $P$. guttatus the two ischial halves were tightly fused with each other at ventro-midline through thick cartilage (pubic ischiadic symphysis, Is) (Figs. 2a, 2b). While in A. boskianus it is articulated antero-medially with each other through pubic ischiadic symphysis (Is) that extended anterio-medially to fuse with a thin rode-like cartilaginous spine (pubic ischiadic, PISS) and posteromedially with a another cartilaginous rode-like process (ischiadic process, ISCS) (Figs.2c,2d).

The ilium (IL) of $P$. guttatus and A. boskianus was a paired curved bony process fused dorsally with ischium and pubis. In $P$. guttatus, the ilium was homogenous rod shape and ends distally by cartilaginous epiphysis (Figs.2a, 2b). While, in A. boskianus, the distal end of ilium was pointed and the cartilaginous epiphysis was no evident (Figs.2c,2d).The skeleton of hind limb of both studied species which consisted of femur, tibia, fibula, metatarsals and digits were calcified. The femur bone (FE) of both $P$. guttatus and $A$. boskianus was more or less, similar in shape to the humerus, possesses a shaft which was quite cylindrical throughout its whole length. The proximal head of the femur articulates 
with the acetabulum of pelvic girdle, while the distal one forms two condyles; the tibular condyle articulates with the tibia and fibular one that articulates with the fibula at the knee. The femur bone in $A$. buskianus is partially calcified, while the femur bone of $P$. gutattus was completely bony structure (Figs.2a,2b, 2c,2d,3). The tibia $(\mathrm{T})$ and fibula $(\mathrm{F})$ of both $P$. guttatus and $A$. boskianus were nearly equal in length, distinctly shorter than the femur, and longer than the radius and ulna. Both the tibia and fibula were partially calcified in A. boskianus, while they were completely bony structure in $P$. gutattus. The tibia was stouter than the fibula and had an expanded head with single broad surface articulated with the most part of the tibial condyle of the femur. The distal part of both fibula and tibia articulated with the tarsal bone at the ankle (Figs.5a, 5b, 5c, 5d, 5e, 5f, 5g, 5h).

The tarsus (TA) was composed of a large proximal bone which articulated proximally with the tibia and fibula and distally with a varying number of distal tarsals. The proximal tarsus (PTA) element of A. boskianus consisted of two fused calcified rudiment bones which may be represented as a fused astragalus and calcaneum and possess on its proximal side two surfaces to articulate with the tibia and fibula (Figs.5e,5f,5g,5h). Meanwhile, the proximal tarsus in $P$. gutattus represented by single large bone with two proximal sockets to articulate with the tibia and fibula and one distal socket to articulates with the fourth distal tarsus (TA4). The distal row of the tarsus consisted of two elements, which represented the third and fourth distal tarsals (Figs. 5a, 5b, 5c, 5d). In $A$. buskianus, the proximal tarsal articulated with the third and fourth distal tarsals. The third distal tarsal articulated with the second and third metatarsus and the fourth distal tarsal articulated with both the fourth and fifth metatarsus (Figs.5e, 5f, 5g, 5h). While, in P.gutattus, the third distal tarsus articulated proximally with the fourth distal tarsus and distally with the first and second metatarsus. The fourth distal tarsus articulated distally with the third, fourth and fifth metatarsus (Figs.5a, 5b, 5c, 5d). In $A$. boskianus the fibular patella and sole sesamoids were missed. The epiphyses of the femur, tibia, fibula, phalanges and distal tarsals were partially calcified. The epiphyses of femur, tibia and fibula were un fused with their shafts (Figs.5e, 5f, 5g, 5h). The metatarsus consisted of five metatarsal bones each of which articulated with one of five phalanges. The longest metatarsus was the second. The typical digital formula of the foot of both $P$. guttatus and A. boskianus was 2. 3. 4. 5. 4 (Fig.5). The digits ended with claws. The last phalanx of $P$. guttatus was produce rode-like process as a continuous structure which supported the terminal pads of each digit (Figs. 5a, 5b, 5c, 5d). 


\section{.Figure Captions}
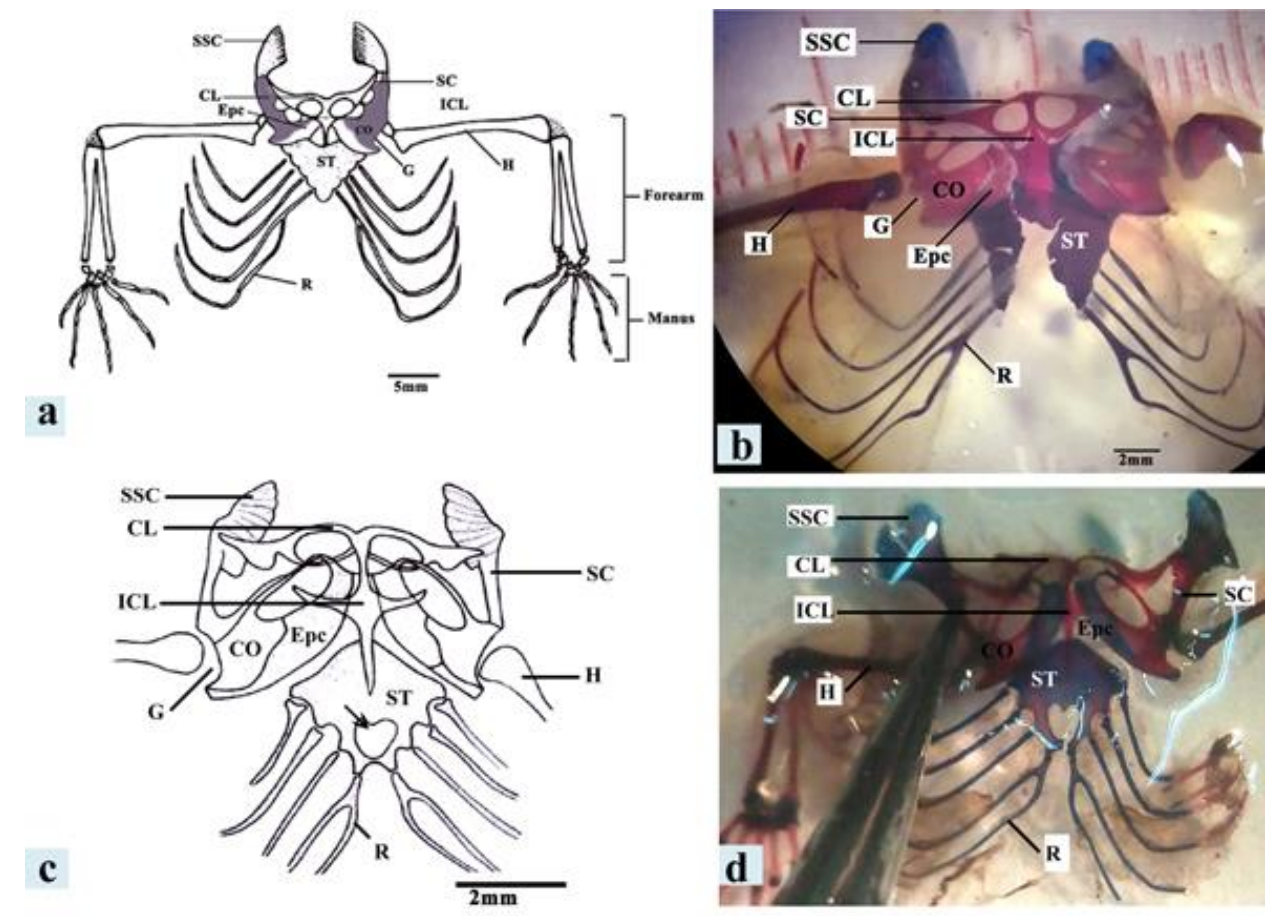

Fig.1

Fig.1. Ventral view of the pectoral girdle; (a\&b) of P. guttatus scale bar $5 \mathrm{~mm}$ and (c\&d) of $A$. boskianus scale bar $2 \mathrm{~mm}$. ("b,d" Stain with Alcian blue-Alizarin red) showing, the paired clavicle (CL), coracoid (CO), epicoraoid (EPC), supra-scapula (SSC), scapulae (SC), unpaired interclavicle (ICL) and sternum (ST) which articulate with ribs (R). Note: glenoid cavity $(\mathrm{G})$ for articulation with proximal end of humerus $(\mathrm{H})$. 


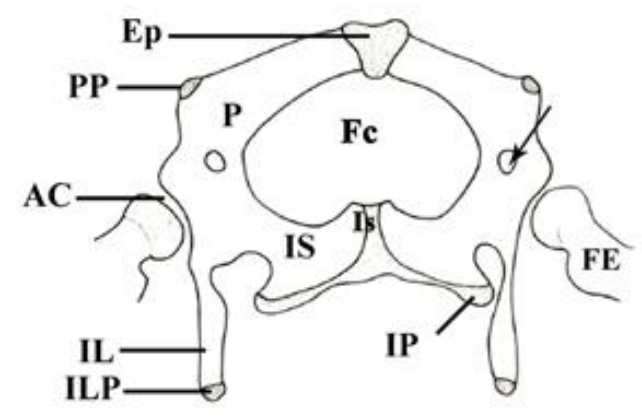

a

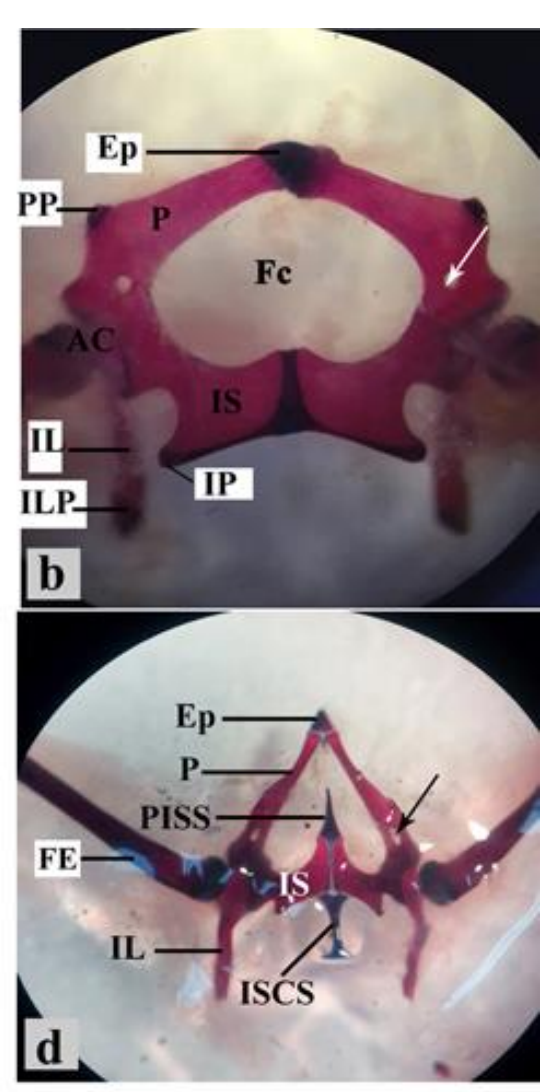

\section{Fig.2}

Fig.2. Ventral view of the pelvic girdle; (a\&b) of P. guttatus and (c\&d) of A. boskianus ("b,d" Stain with Alcian blue-Alizarin red) showing pubis (P), ilium (IL), ischium (IS), ischio-pubic fenestra (Fc), ischiadic process (IP), epipubic (Ep), prepubic (PP), obturator foramen (arrow), pubic ischiadic symphysis (Is), pubic ischiadic (PISS), ischiadic process (ISCS) and acetabulum (AC) for articulation with proximal end of femur (FE).scale bar $2 \mathrm{~mm}$. 
Ali et al.

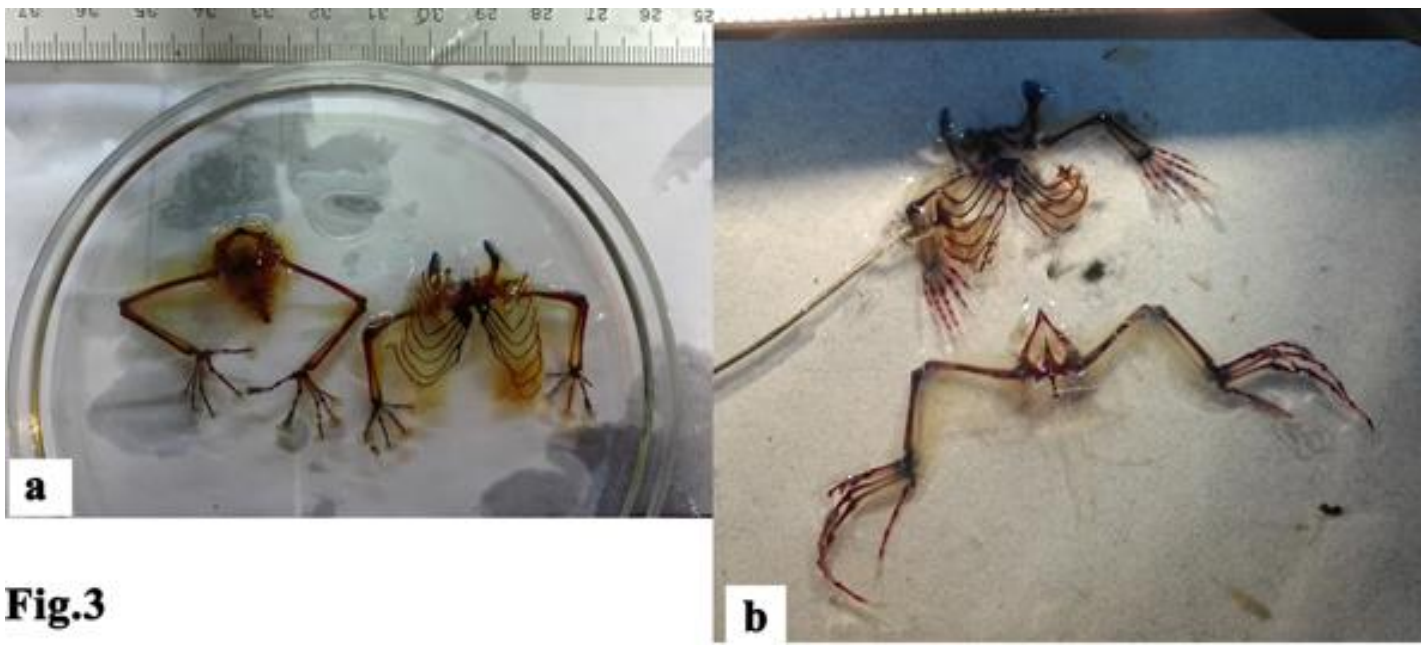

Fig.3. Photomacrograph of the appendicular skeleton of (a) P. guttatus (b) A. boskianus (Stained with Alcian blue-Alizarin red) showing the limbs posture of limbs of both studied species.
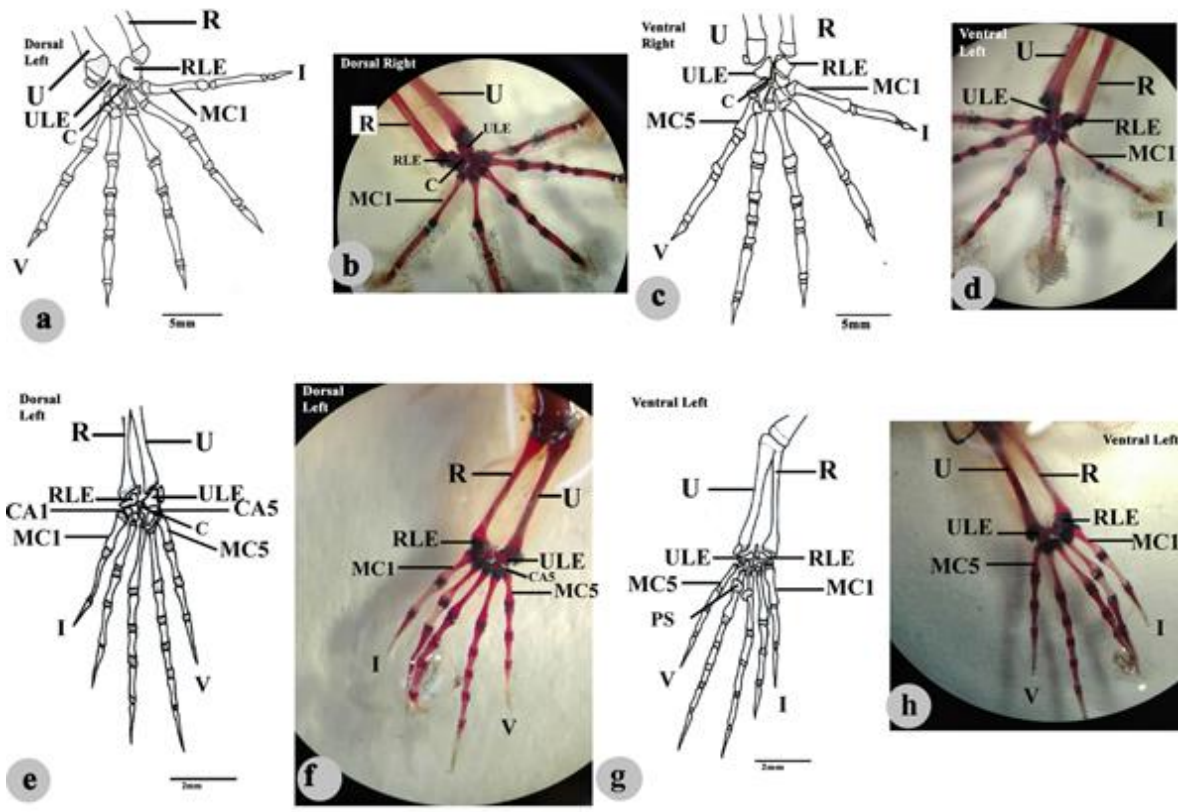

Fig.4

Fig.4. Fore limbs; (a\&b; dorsal view of left and right forelimb, (c\&d) ventral view of left and right fore-limb of $P$. guttatus) scale bar 5mm, (e\&f; dorsal view of left forelimb and $(\mathrm{g} \& \mathrm{~h})$ ventral view of left forelimb of A. boskianus) scale bar $2 \mathrm{~mm}$ ("b,d,f,h" Stain with Alcian blue-Alizarin red) showing radius (R), ulna (U), radiale (RLE), centrale (C) and ulnare (ULE) and palmar sesamoids (PS) at ventral view. 


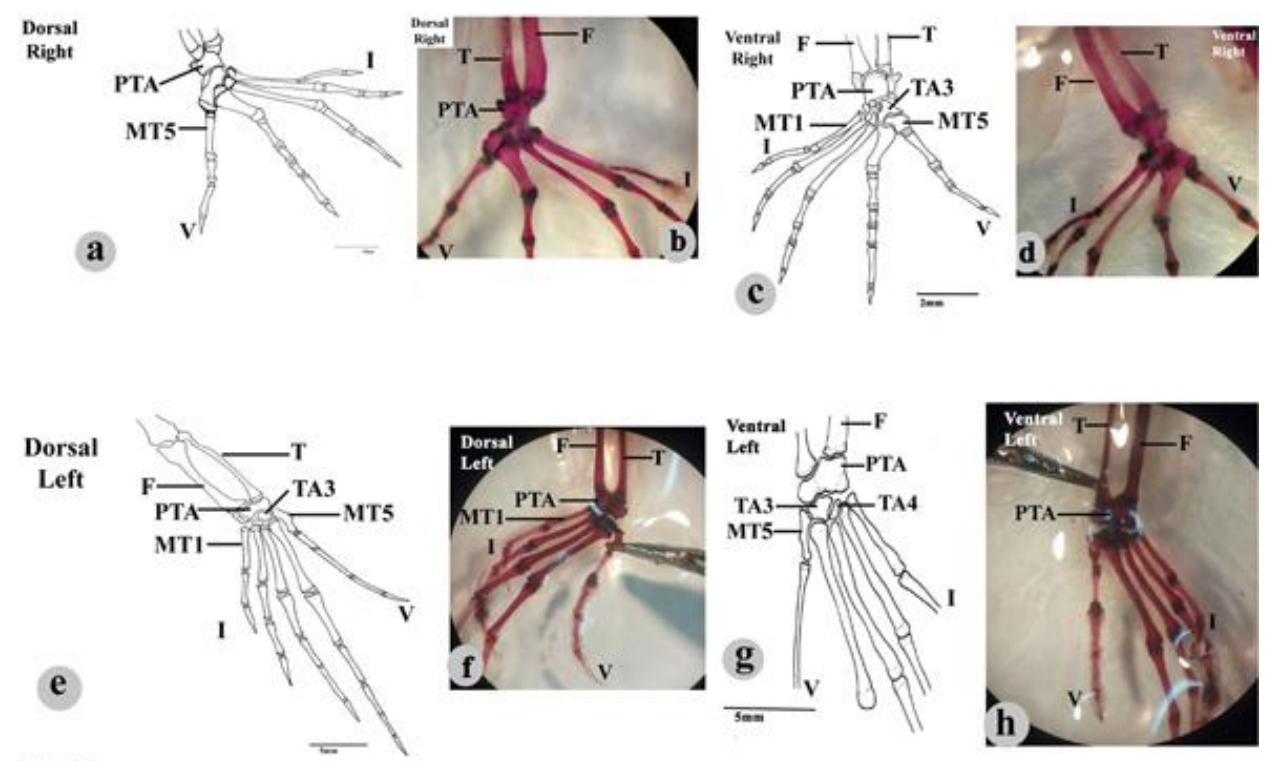

Fig.5

Fig.5. Hind limbs; (a\&b; dorsal view of right hind limb and (c\&d) ventral view of right hind limb of $P$. guttatus) scale bar $2 \mathrm{~mm}$ and (e\&f; dorsal view of left hind limb and ( $\mathrm{g} \& \mathrm{~h}$ ) ventral view of left forelimb of A. boskianus) scale bar 5mm ("b,d,f,g" Stain with Alcian blue-Alizarin red) showing tibia (T) and fibula (F), proximal tarsus (PTA), third, fourth distal tarsus (TA3,TA4) and metatarsus (MT).

\section{Osteometric characters of the fore and hind limbs.}

In both studied species, the limbs length exhibited differences between all the investigated specimens of the two species. The fore and hind limbs of $A$. boskianus were shorter in length in comparison with the fore and hind limbs of
P. guttatus (Figs.6,7,8,9 and table 1,2). There was relationship between fore and hind limbs lengths in all the investigated specimens of the two species. The hind limb of A. boskianus was longer than the fore limb, while in P. guttatus the fore limb was relatively of the same length of the hind limb (Figs.8,9 and table 1,2). 
Ali et al.

Table1. The osteometric characters of the fore limb of two examined squamates species. The basic statistics (mean $\pm \mathrm{SD}$ )

\begin{tabular}{|l|c|c|}
\hline \multirow{2}{*}{ Osteometric characters } & A. boskianus & $P$. guttatus \\
\cline { 2 - 3 } & $\mathrm{X} \pm \mathrm{SD}$ & $\mathrm{X} \pm \mathrm{SD}$ \\
\hline Fore limb length (FOL) & $17.18 \pm 0.46$ & $34.92 \pm 0.88$ \\
\hline Humerus length (HL) & $5.82 \pm 0.15$ & $14.55 \pm 0.38$ \\
\hline Ulnar length (UL) & $4.65 \pm 0.19$ & $13.72 \pm 0.23$ \\
\hline Redial length (RL) & $3.95 \pm 0.28$ & $11.7 \pm 0.34$ \\
\hline Manus length (MAL) & $7.42 \pm 0.24$ & $8.73 \pm 0.26$ \\
\hline DIM & $2.82 \pm 0.19$ & $2.75 \pm 0.18$ \\
\hline DIIM & $3.7 \pm 0.14$ & $3.7 \pm 0.31$ \\
\hline DIIIM & $4.5 \pm 0.28$ & $5.4 \pm 0.27$ \\
\hline DIVM & $5.18 \pm 0.19$ & $4.9 \pm 0.14$ \\
\hline DVM & $3.65 \pm 0.19$ & $2.9 \pm 0.14$ \\
\hline
\end{tabular}

Table2. The osteometric characters of the hind limb of two examined squamates species. The basic statistics (mean $\pm \mathrm{SD}$ )

\begin{tabular}{|l|c|c|}
\hline \multirow{2}{*}{ Osteometric characters } & A. boskianus & P. guttatus \\
\cline { 2 - 3 } & $\mathrm{X} \pm \mathrm{SD}$ & $\mathrm{X} \pm \mathrm{SD}$ \\
\hline Hind limb length (HIL) & $27.93 \pm 0.61$ & $41.83 \pm 0.49$ \\
\hline Femur length (FEL) & $8.6 \pm 0.39$ & $15.77 \pm 0.22$ \\
\hline Fibula length (FL) & $6.17 \pm 0.22$ & $13.5 \pm 0.35$ \\
\hline Tibia length (tL) & $6.15 \pm 0.37$ & $13.73 \pm 0.29$ \\
\hline Pes length (PEL) & $13.18 \pm 0.22$ & $12.33 \pm 0.45$ \\
\hline DIP & $2.93 \pm 0.12$ & $2.6 \pm 0.29$ \\
\hline DIIP & $5.67 \pm 0.29$ & $4.02 \pm 0.36$ \\
\hline DIIIP & $6.42 \pm 0.32$ & $4.67 \pm 0.33$ \\
\hline DIVP & $7.62 \pm 0.31$ & $6.82 \pm 0.23$ \\
\hline DVP & $6.07 \pm 0.30$ & $4.43 \pm 0.48$ \\
\hline
\end{tabular}



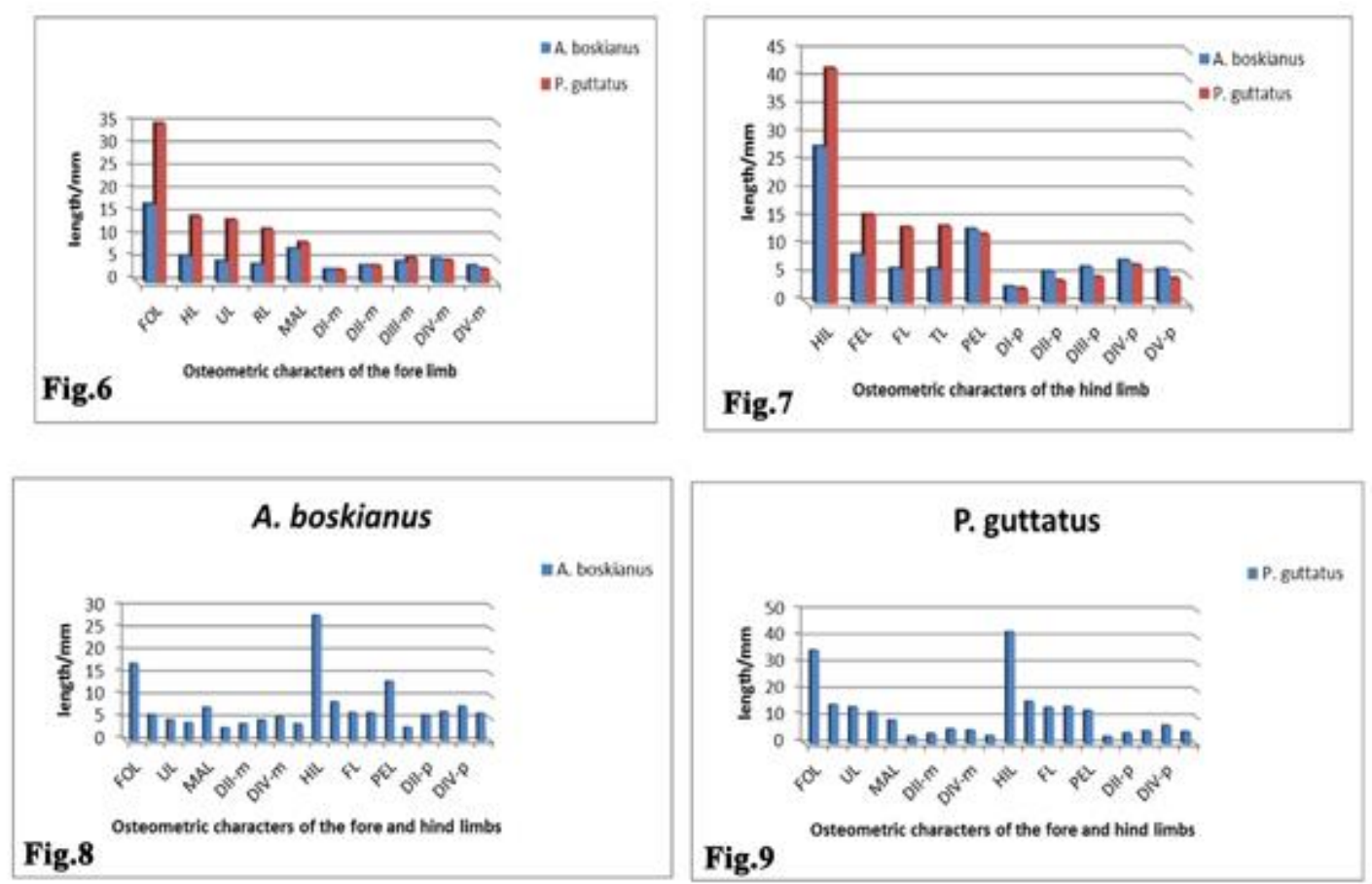

Fig.6. Histogram shows the osteometric characters of the fore limb of A. boskianus and $P$. guttatus

Fig.7. Histogram shows the osteometric characters of the hind limb of A. boskianus and $P$. guttatus

Fig.8. Histogram shows the osteometric characters of the fore and hind limbs of $A$. boskianus

Fig.9. Histogram shows the osteometric characters of the fore and hind limbs of $P$. guttatus

\section{Discussion}

The skeletal elements of both the pectoral and pelvic girdles of both studied species exhibited great similarity with little variation in the degree of calcification and fusion of some elements. In P. guttatus, the pectoral girdle was characterized by the presence of the paired interclavicles which was dagger in shape and partially fused with clavicles, while it was cruciform and completely fused with the clavicles in A. boskianus. In P. guttatus, the epicoraoid was well developed, broad bony plates and fused with the interclavicles. While, in A. boskianus it was narrow cartilaginous, plates and overlap in the mid-line. Moreover, in $P$. gutattus two fenestrae appeared within coracoid and scapula with un-fenestrated cartilaginous sternum, while one fenestra appeared in both coracoid and sternum of A. boskianus. On the other hand, the pelvic girdle of $P$. guttatus exhibited complete fusions between the pubis, ischium and ilium, while in $A$. boskianus the pubis articulated posteromedially with the ischium. Similar observations were recorded in Hemidactylus turcicus, Acanthodactylus scutellatus ${ }^{[5]}$, Scincids ${ }^{[6]}$. But, in gecko in Australia, the elements of 
pectoral girdle were fully calcified and scapula and coracoids are fused ${ }^{[7]}$.

Most noticeably in the pectoral and pelvic girdle of $P$. guttatus, increased degree of calcification was detected in most skeletal elements of both girdles and fusion between epicoracoid and interclavicle and un-fenestered sternum in the pectoral girdle as well as pubis, ischium and ilium in the pelvic girdle. That fusion between skeletal elements of both girdles in P. guttatus allowed welldeveloped axial musculature to produce lateral-sequence gait in which the central of mass never left the triangular of support established at any one moment during a gait cycle by three of the four limbs ${ }^{[8]}$, during lateral gait of gecko, the resulting forces of retracting limbs directly toward the midline of both girdles, thereby must strengthened to receive this producer forces. Meanwhile, A. boskianus exhibited flexible articulation between skeletal elements of the girdles to give the limbs great freedom of movement. A. boskianus is one of bipedial locomotion species living in open sandy or rocky areas. To adjust this environment it must increase in speed or posture to escape from predators. Similar conclusion was recorder by ElBakry et al. ${ }^{[5]}$.

Moreover, in A. boskianus the pubis and ischiadic symphysis,epipubic and ischiadic process were well developed and cartilaginous while, in $P$. guttatus that process were not evident and the pubis and ischiadic symphysis were partially calcified. These results disagree with those mentioned in Acanthodactylus scutellatus by El-Bakry et al. ${ }^{[5]}$ reported that the epipubis and ischiadic symphysis were partially calcified. Stephenson [7] reported that these two rudiments of the juvenile forms may not be, or may be only slightly calcified, although in older specimens, the epipubis became strongly calcified as the ischiadic symphysis region. These two elements were fully cartilaginous in the scincids Mabuya aurata and Mabuya quinquetaeniata and in several geckos ${ }^{[6,9]}$. However, in the present study, it was observed that the acetabulum and glenoid fossae of $P$. gutattus and $A$. boskianus were shifted postero-ventrally that follow the inwards shift in limb posture. The placement of forelimbs directly beneath the scapula shifted mechanically forces away the midline to the scapula. This gives the scapula a greater role in locomotion and weight bearing, such similar feature are established in therapsids ${ }^{[8]}$. In $P$. gutattus and $A$. boskianus, the forelimbs posture was near the horizontal plane, while the hind limbs in vertical plane. The crus moved near vertical the plane and resulted in a force with potential posterior and lateral components and the femur back swing parallel to the body long axis, perpendicular to the pedal long axis. Rewcastle ${ }^{[10]}$ and Brinkman ${ }^{11]}$ concluded that the pes acted as an additional limb lever. Zaff $\boldsymbol{e t} \boldsymbol{a l} .{ }^{[12]}$ noted that in the climber, the fore limbs became more important in the production of locomotor thrust and the maintenance of grip and documented more powerful shoulder retractor muscles in gecko and higher flexion moments across the elbow, preventing the animal from falling backwards when on vertical surfaces. These observations are parallel with the qualitative observations of Arnold ${ }^{[13]}$.Moreover, in P. gutattus both the fore and hind limbs were relatively equal in length, while the fore limb in A. boskianus was greatly shorter than the hind limb. Ground-dwellers had relatively long hind limb, with high tibia: femur ratios, and relatively short fore limbs. Climbers had 


\section{Comparative Functional Analysis of the Anatomy......}

relatively short limbs, with low tibia: femur ratios and equally long hind and fore limbs ${ }^{[14]}$. Based on general biomechanical principles it can be predicted that level runners had longer limbs, moving more sagittaly to increase the stride length and elevated the body from the ground to reduce friction ${ }^{[15,16,17]}$. Climbers on the other hand, should be fit from shorter limbs and a more sprawling gait to keep the centre of mass as close to the substrate as possible ${ }^{[18-19]}$. In the present study $P$. gutattus, the limbs ended by adhesion pads. This finding is confirmed by results of Saber et al. [20] who stated that both limbs of $P$. gutattus ended by adhesion pads that consisted of cluster of setae while, limbs of $A$. Boskianus ended by pointed claws. In climbing gecko, the setae employed a distinctive feature in their locomotion, these setae holding the pad perpendicular to the surface.

In conclusion $P$. gutattus is considered as a one of climbers species has stout appendicular skeleton to support the climbing process on the vertical surface with relatively short limbs, while $A$. Boskianus is ground-dwellers and also good climbers, used their claws to walk and climbing on rocky areas. These abilities of locomotion depend on the flexibility of skeletal elements of its appendicular skeleton.

\section{References}

1. Brana F (2003): Morphological correlates of burst speed and field movement patterns: the behavioural adjustment of locomotion in wall lizards (Podarcis muralis). Biol. J. Linn. Soc., 80: 135-146.

2. Nauwelaerts $S$ and Aerts $P$ (2002): Two distinct gait types in swimming frogs. J. Zool. Lond., 258: 183-188.

3. Glasheen $\mathbf{J} \mathbf{W}$ and McMahon $\mathbf{T} A$ (1996): Size-dependence of water-running abilityin basilisk lizards (Basiliscus basiliscus). J. of Exp. Biol., 199: 26112618.

4. Losos J B, Warheit K I and Schoener T W (1997): Adaptive differentiation following experimental island colonization in Anolis lizards. Nature, 387: 70-73.

5. El-Bakry A M, Abdeen A M and AboEleneen R E (2013): Comparative study of the osteology and locomotion of some reptilian species. International J. of Biol. and Biol. Sci., 2(3): 40-58.

6. Mohammed M B H (1990): Some observations on the classification pattern in the appendicular skeleton during the growth of the skink Mabuya (Scincidae, Reptilia). J. Egypt. Ger. Soc. Zool., 1: 197212.

7. Stephenson N G (1960): The comparative osteology of Australian geckos and its bearing on their morphological status. Zool. J. Linn. Soc., 44: 278- 299.

8. Kardong K V (2009): Vertebrates: Comparative Anatomy, Function, Evolution. $7^{\text {th }}$ ed., Boston: McGraw-Hill, Amazon.London P.816.

9. Mohammed M B H (1988): Calcification pattern in the appendicular skeleton of certain geckos (Gekkonidae Reptilia). Ans. Zool. India., 26: 1-20.

10. Rewcastle S C (1983): Fundamental adaptations in the lacertilian hind-limb: a partial analysis of the sprawling limb posture and gait. Copeia, 1983: 467-487.

11. Brinkman D (1981): The hind limb step cycle of iguana primitive reptiles. J. Zool. Lond., 181: 91-103.

12. Zaff A, Herrel $A$, Aerts $P$ and De Vree F (1999): Morphology and morphometrics of the appendicular musculature in geckos with different locomotor habitat. Zool. Morphol., 119: 9-22.

13. Arnold E N (1998): Structural niche, limb morphology and locomotion in lacertid lizards (Squamata: Lacertidae), a preliminary survey. Bull. Brit. Mus. Nat. Hist. Lond. Zool., 64: 63-89. 
14. Zaaf $A$ and Van Damme $R$ (2001): Limb proportions in climbing and grounddwelling geckos (Lepidosauria, Gekkonidae): a phylogenetically informed analysis. Zoomorphol., 121: 45-53.

15. Rewcastle S C (1981): Stance and gait in tetrapods: an evolutionary scenario. Symp. Zool. Soc. Lond, 48:239-267.

16. Miles D B, Fitzgerald $L A$ and Snell $H$ L (1995): Morphological correlates of locomotor performance in hatchling Amblyrhynchus cristatus. Oecologia, 103:261-264.

17. Bauer A M, Russell A P and Powell G L (1996): The evolution of locomotor morphology in Rhoptropus (Squamata: Gekkonidae): functional and phylogenetic considerations. Afr. J. Herpetol., 45: 830 .
18. Peterson J A (1984): The locomotion of Chamaeleo (Reptilia: Sauria) with particular reference to the fore limb. J. Zool., 202: 1-42.

19. Van damme $R$, Aerts $P$ and Van hooydonck B (1997): No trade-off between sprinting and climbing in two populations of the lizard Podarcis hispanica (Reptilia: Lacertidae). Biol. J. Linn. Soc., 60: 493-503.

20. Saber S A, EISalkh B A, Gadel-Rab A G, Mahmoud F A, El Dahshan A A and Gewily D I (2018): Comparative and functional study of integumentary system of two different reptiles: adaptation to their different modes of life. The Egyptian J. of Hospital Medicine, 73 (6): 6802-6811. 